\title{
B/L Basal Ganglia Lesions in a Child Leading to a Diagnosis of Glucose-6-Phosphate Dehydrogenase Deficiency
}

\author{
Nidhi Prabhakar Chirag K. Ahuja Niranjan Khandelwal \\ Department of Radiodiagnosis and Imaging, PGIMER, Chandigarh, India
}

\section{Keywords}

Glucose-6-phosphate dehydrogenase - Basal ganglia ·

Kernicterus · Child

\begin{abstract}
Bilateral basal ganglia lesions are a common non-specific finding seen in many diseases. One of the differential diagnoses for it, in a child, is kernicterus occurring due to hyperbilirubinemia. Glucose-6-phosphate dehydrogenase (G6PD) deficiency is a common cause of severe hyperbilirubinemia. A 1-year old child presented to the hospital with history of generalized dystonia in the previous 3 days. MRI showed evidence of symmetrical lesions in bilateral globus pallidus, which were hyperintense on T2/FLAIR and isointense on T1. Patient's blood test revealed G6PD deficiency. Hence, a diagnosis of G6PD deficiency leading to kernicterus was made. In a child, the diseases that may affect the basal ganglia symmetrically and bilaterally include kernicterus, hypoxia, carbon monoxide poisoning, hypoglycemia, inherited metabolic and dysmyelinating disorders like Leigh disorder, Canavan and Krabbe, Neurofibromatosis, Herpes encephalitis, congenital HIV infection, manganese poisoning and extrapontine myelinolysis. Important causes of kernicterus are Rh incompatibility, $A B O$ incompatibility, sepsis, hemolytic anaemia and G6PD deficiency. G6PD deficiency leading to
\end{abstract}

kernicterus should be considered a differential diagnosis of bilateral basal ganglia lesions in children. Proper elicitation of history with appropriate blood biochemical tests will help in arriving at a proper diagnosis.

(c) 2017 S. Karger AG, Basel

\section{Introduction}

Bilateral basal ganglia lesions are a common non-specific finding seen in many diseases [1]. One of the differential diagnoses for it, in a child, is kernicterus occurring due to hyperbilirubinemia. Glucose-6-phosphate dehydrogenase (G6PD) deficiency is a common cause of severe hyperbilirubinemia [2]. In this article, we present a case of a child who showed bilateral basal ganglia lesions on MRI, due to kernicterus and on investigation, and was diagnosed to be a case of G6PD deficiency.

\section{Case Report}

A 1-year old child presented to the hospital with history of generalized dystonia for the last 3 days. MRI showed evidence of symmetrical lesions in bilateral globus pallidus, which were hyperintense on T2/FLAIR and isointense on T1 (Fig. 1a, b). There was no contrast enhancement seen in them. There was no diffusion restriction.

\section{KARGER}

(c) 2017 S. Karger AG, Basel

E-Mail karger@karger.com

www.karger.com/aon
Dr. Chirag K. Ahuja

Department of Radiodiagnosis and Imaging

PGIMER, Sector 12

Chandigarh (India)

E-Mail chiragkahuja@ rediffmail.com 

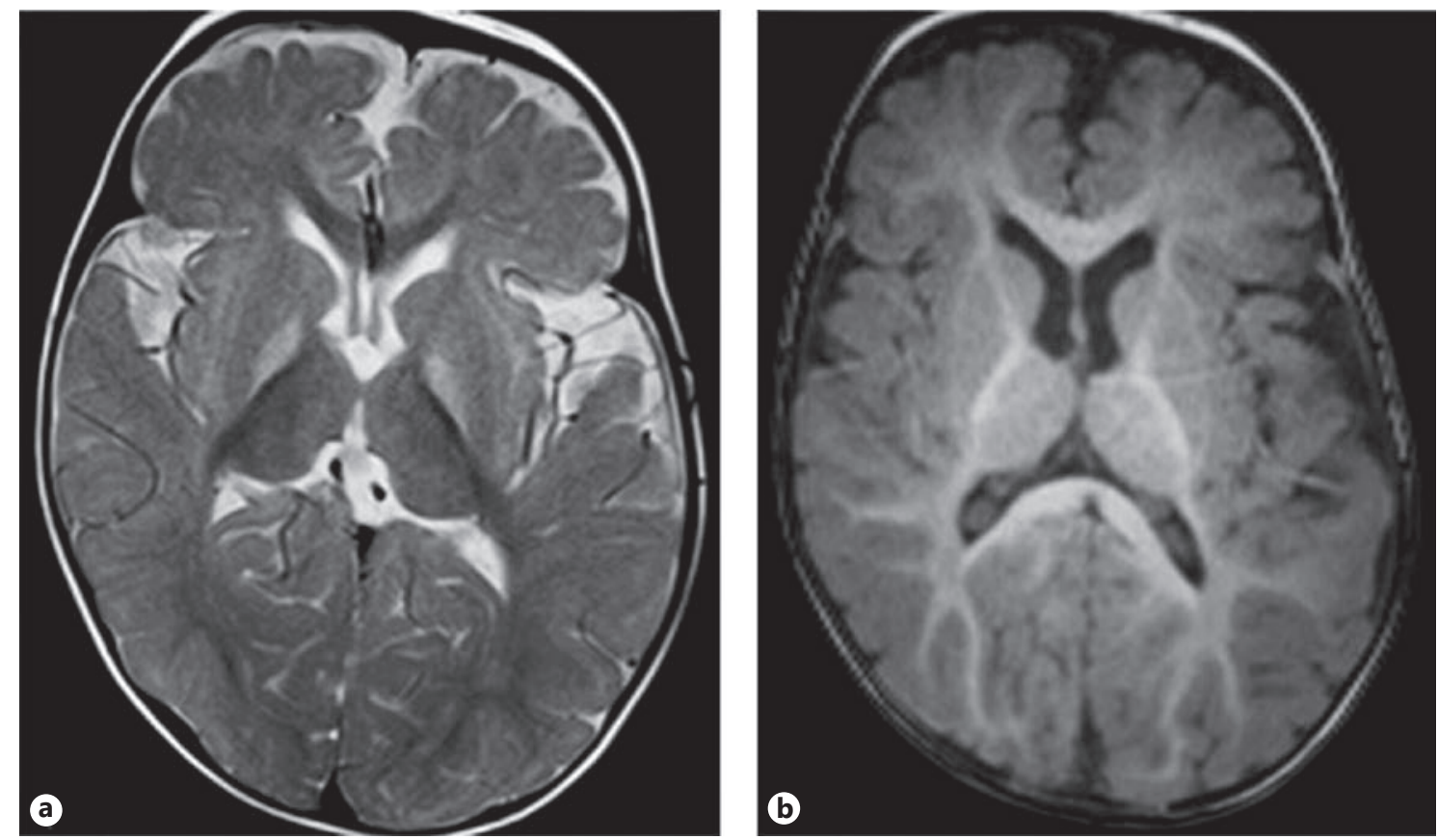

Fig. 1. Axial MRI images of brain of 1-year old child showing symmetrical lesions in bilateral globus pallidus. a T2-weighted images showing lesions are hyperintense. b T1-weighted images showing lesions are isointense.

Parents of the patient were asked about the birth and neonatal history of the patient. The child was born at term and with normal vaginal delivery. He had spontaneous cry at birth. However, patient had jaundice on the 10th day with peak total serum bilirubin levels of $24 \mathrm{mg} / \mathrm{dL}$. Exchange transfusion brought down the total serum bilirubin level to normal. Patient was discharged after 5 days. No other work up was done.

The patient's blood test revealed G6PD deficiency. Hence, a diagnosis of G6PD deficiency leading to kernicterus was established.

\section{Discussion}

The term basal ganglia includes caudate and lentiform nuclei. Lentiform nuclei consist of globus pallidus and putamen. Basal ganglia, substantia nigra and subthalamic nuclei belong to the extrapyramidal system. There are a number of diseases, which may affect bilateral basal ganglia. MRI characteristics of the basal ganglia lesions, along with the age and clinical symptoms of the patient, aid in arriving at a diagnosis.

In a child, the diseases that may affect the basal ganglia symmetrically and bilaterally include kernicterus, hypoxia, carbon monoxide poisoning, hypoglycemia, inherited metabolic and dysmyelinating disorders like Leigh disorder, Canavan and Krabbe, Neurofibromatosis, Herpes encephalitis, congenital HIV infection, manganese poisoning and extrapontine myelinolysis [1].

Kernicterus is an important cause and should be considered in the differential diagnosis. "Kernicterus" word was coined by Schmorl in 1903, on discovering the pathologic finding of canary yellow staining of basal ganglia in infants who had neonatal jaundice. It is caused due to the deposition of unconjugated bilirubin within the brain [3]. The most commonly affected areas are the basal ganglia (predominantly globus pallidus), subthalamic nuclei, dentate nuclei, hippocampus (sector H2, H3), cerebellar vermis and cranial nerve nuclei (oculomotor, vestibular and cochlear). Periventricular infarcts may also be present. However, cortex is spared [4]. On MRI, this deposition is seen as hyperintensity on T1 in acute cases and T2 hyperintensity in chronic lesions. Thalami are spared in kernicterus. This may help in differentiating between hypoxia and metabolic disorders, which commonly affect the thalami also.

Important causes of kernicterus are Rh incompatibility, ABO incompatibility, sepsis, hemolytic anaemia and G6PD deficiency [5]. G6PD deficiency was discovered in 1957 [6]. G6PD enzyme is important in the glycolytic cycle (Embdem Meyerhof), which converts glucose to lactate, with production of ATP. The first step in the cycle is catalyzed by this enzyme, with production of NADPH. 
NADPH leads to reduction of glutathione to GSH and methemoglobin to oxyhemoglobin. Deficient G6PD enzyme leads to reduced production of GSH and ATP. GSH is a substrate of Glutathione peroxidase, which protects cell from toxic effects of $\mathrm{H}_{2} \mathrm{O}_{2}$, which is produced by some drugs [7]. Some drugs also lead to the damage of RBC cell membrane, with increased permeability to cations, which is compensated by an ATP-dependent transport mechanism. If ATP is deficient, there is failure of compensation, loss of osmotic equilibrium and hemolysis [8]. The increased hemolysis leads to increased unconjugated bilirubin and consequently kernicterus.

G6PD deficiency disease is gender linked. The gene for this is present on $\mathrm{X}$ chromosome. It usually gets manifested in hemiazgous males, homozygous females and a few heterozygous females.

About 400 million people are affected worldwide, with increased prevalence in Africa, Middle East, tropical and subtropical Asia, Mediterranean, Papua and New Guinea [9]. About 24 million children are born in India annually and 390,000 out of them are affected by this disease [10].

G6PD deficiency usually manifests in newborn as jaundice. Jaundice occurs after $24-48 \mathrm{~h}$ of neonatal life. Some even manifest in $3 \mathrm{rd}$ to 4 th week. If jaundice leads to kernicterus, patient usually presents with athetoid cerebral palsy, speech or hearing impairment. In older pa- tients, it presents as acute hemolytic crisis, on taking drugs like chloramphenicol, primaquin, chloroquin, sulphonamides, chemicals like naphthalene and henna and exposure to viral and bacterial infections.

Prevention of kernicterus is important in neonates with hyperbilirubinemia. Phototherapy and exchange transfusion are used to bring down the bilirubin levels. It is important to avoid drugs that may predispose to hemolytic crisis in patients of G6PD deficiency.

In conclusion, G6PD deficiency resulting in kernicterus could be considered a differential diagnosis of bilateral basal ganglia lesions in children. Detailed history with appropriate blood biochemical tests may help in arriving at a proper diagnosis.

\section{Disclosure Statement}

Authors declare no conflict of interest. The manuscript complies with ICMJE.

\section{Authorship Contributions}

Imaging was done by Dr. Chirag Ahuja. Manuscript preparation was done by Dr. Nidhi Prabhakar. Manuscript Editing was done by Dr. Chirag Ahuja, Dr. Niranjan Khandelwal.

\section{References}

Bekiesinska-Figatowska M, Mierzewska $\mathrm{H}$, Jurkiewicz E: Basal ganglia lesions in children and adults. Eur J Radiol 2013;82:837-849.

2 Weng YH, Chiu YW: Clinical characteristics of G6PD deficiency in infants with marked hyperbilirubinemia. J Pediatr Hematol Oncol 2010;32:11-14.

3 Schmorl G: Zur Kenntnis des ikterus neonaturum, insbesondere der dabei auftretenden gehirnveranderungen. Verh Dtsch Ges Pathol 1904;6:109-115.

-4 Turkel SB, Miller CA, Guttenberg ME, Moynes DR, Godgman JE; A clinical patho- logic reappraisal of kernicterus. Pediatrics 1982;69:267-272

5 Parashari UC, Singh R, Yadav R, Aga P: Changes in the globus pallidus in chronic kernicterus. J Pediatr Neurosci 2009;4:117-119.

-6 Alving AS, Carson PE, Flanagan CL, Ickes CE: Enzymatic deficiency in primaquinesensitive erythrocytes. Science 1956;124:484485.

7 Cohen G, Hochstein P: Glutathione peroxidase: the major pathway of peroxide detoxification of erythrocytes. Biochemistry 1963;2: $1420-1428$.
8 Mohler DN, Crockett CL: Hereditary hemolytic disease secondary to glucose-Bphosphate dehydrogenase deficiency: report of three cases with special emphasis on AlT metabolism. Blood 1964;23:427444.

9 Nair H: Neonatal screening program for G6PD deficiency in india: need and feasibility. Indian Pediatr 2009;46:1045-1049.

10 Verma IC, Bijarnia S: The burden of genetic disorders in India and a framework for community control. Community Genet 2002;5: 192-196. 